\title{
Validation of a global quantitative analysis methodology of tryptophan metabolites in mice using LC-MS
}

Antoine Lefèvre ${ }^{\mathrm{a}}$, Sylvie Mavel ${ }^{\mathrm{a}^{*}}$, Lydie Nadal-Desbarats ${ }^{\mathrm{a}}$, Laurent Galineau ${ }^{\mathrm{a}}$, Sylvie Attucci ${ }^{\mathrm{a}}$, Diane Dufour ${ }^{\mathrm{a}, \mathrm{b}}$, Harry Sokol ${ }^{\mathrm{c}, \mathrm{d}}$, Patrick Emond ${ }^{\mathrm{a}, \mathrm{b}}$

${ }^{a}$ UMR 1253, iBrain. Université de Tours, Inserm, Tours, France.

${ }^{b}$ CHRU de Tours, Service de Médecine Nucléaire In Vitro, Tours, France

c Sorbonne Université, Ecole normale supérieure, PSL Research University, CNRS, INSERM, AP-HP, Gastroenterology Department, Hôpital Saint-Antoine, Laboratoire de biomolécules, LBM, 184 rue du Faubourg Saint-Antoine, Paris 75005, France

${ }^{\mathrm{d}}$ INRA, UMR1319 Micalis \& AgroParisTech, Jouy en Josas, France

Keywords : tryptophan pathway; kynurenine pathway; liver matrix; cecal and intestinal contents; validation methodology 


\section{ABSTRACT:}

In this study, we validated a method for quantifying 20 tryptophan (Trp) catabolites by liquid chromatography coupled with high resolution mass spectrometry (LC-HRMS) in 4 different matrices (urine, serum, intestinal contents and liver). The detection limit for all metabolites ranged between 0.015 and $11.25 \mathrm{nmol} / \mathrm{L}$ and the dynamic range of the calibration curves were adjusted to allow quantification of metabolites at endogenous levels. Matrix effects were evaluated using isotope labeled internal standards. Reproducibility in the 4 matrices was characterized by $\mathrm{CV}=6.2 \%$ with an accuracy of $6.6 \%$. Our method has been applied to the determination and quantification of 20 metabolites concentrations in 5 different mouse compartments (plus cecal contents). Our results show that our approach allows for a global exploration of the Trp metabolism by quantifying a large number of Trp metabolites, at the individual level by multi-matrix approach.

Abbreviations: 3-Hydroxyanthranilic acid, 3-HAA; 3-Hydroxykynurenine, 3-HK; 5Hydroxyindole acetic acid, 5-HIAA; 5-Hydroxytryptophan, 5-HTP; Indole-3-acetamide, IAM; Indole-3-acetic acid, IAA; Indole-3-carboxaldehyde, I-3CA; Indole-3-lactic acid. ILA; Indoxyl sulfate, 3-IS; Kynurenic acid, KA; Kynurenine, Kyn; N-Acetylserotonin. N-AS; Quinolinic acid, QA; Serotonin, 5-HT; Tryptamine, TA; Tryptophan, Trp; Xanthurenic acid, XA. 


\section{Introduction}

Tryptophan (Trp) is an essential amino acid that is built around an indole nucleus and has the highest molecular weight of all amino acids. Trp cannot be synthesized from other compounds by the human body. However, some microorganisms of the intestinal microbiota have been shown to produce this amino acid [1]. Although Trp is the least abundant amino acid in cellular proteins, it feeds several metabolic pathways both in the host and in the complex community of microorganisms that live in the digestive tract [2]. About $90 \%$ of Trp is used by the kynurenine pathway [3] and this metabolism is regulated by the expression of indoleamine dioxygenase (IDO1) found in all tissues and by tryptophan-2,3-dioxygenase (TDO) found exclusively in the liver [4]. Intestinal microbiota has also been shown to modulate the kynurenine pathway, thereby influencing the physiology of the digestive tract and of the central nervous system. Indeed, the kynurenine pathway is thought to play a key role in the modulation of neurotransmission and immune functions [3]. Consequently, fluctuations of the kynurenine pathway are associated with many psychiatric and gastrointestinal disorders.

A second metabolic pathway of Trp leads to the production of 5-hydroxytryptamine (serotonin, 5-HT). Serotonin is a neurotransmitter produced in the brain stem via tryptophan hydroxylase 2. However, $90 \%$ of the serotonin is peripherally produced by intestinal enterochromaffin cells via tryptophan hydroxylase 1 (TrpH1). Although serotonin does not cross the blood-brain barrier, 5-hydroxytryptophan which is produced by the action of TrpH1 on Trp, readily crosses the blood brain barrier to reach the brain where it is converted to serotonin (5-HT). Serotonin triggers many functions both in the gastrointestinal tract and the brain, and is involved in a wide range of human physiological functions by activating specific

\section{5-HT receptors [5].}

Finally, Trp can be metabolized into indole derivatives, mostly by intestinal microorganisms. These indole metabolites are aryl hydrocarbon receptor (AhR) ligands, and are considered a 
key component of the immune response, although their functions are not fully understood [6, 7] .

Due to the multitude of physiological functions involving Trp metabolites, disruptions of its metabolism are associated with many pathologies such as inflammatory bowel disease (IBD), irritable bowel syndrome (IBS), metabolic disorders (diabetes, obesity, non-alcoholic fatty liver disease, insulin resistance and atherosclerosis) and neuropsychiatric affections, including anxiety, depression and autism (see [8] for a recent review).

Despite its importance, a detailed knowledge of the activities of the different pathways of Trp metabolism with respect to compartments or physiopathological conditions remains to be investigated. Methods capable of quantifying all the catabolites of Trp in different key compartments should make it possible to identify, confirm and validate key biomarkers resulting from this metabolism.

A number of studies have reported methods [9-13] for assaying certain Trp metabolites but were all dedicated to discrete physiological compartments. To broaden Trp metabolite assaying from a separate to a connected approach, we sought to develop a new method for the simultaneous quantification of Trp metabolites in different physiological compartments. This has been achieved using liquid chromatography coupled with high resolution mass spectrometry (LC-HRMS). The method employed a stable isotope labeled (SIL) internal standards strategy, and was validated on 4 distinct physiological matrices (serum, urine, intestinal contents and liver) and applied to the quantification of metabolites in samples coming from mice on 5 matrices (plus cecal contents). Our findings provide an overview of peripheral Trp metabolism (see supplementary material, Fig. A1) at an organism level. 


\section{Materials and methods}

\subsection{Reagents, and solutions}

All tryptophan metabolites (see supplementary material, Fig. A1) were purchased from Sigma Aldrich. All SILs 5-Hydroxyindole acetic acid- $\mathrm{d}_{5}$, Serotonin- $\mathrm{d}_{4}$, Indole-3-acetic acid$\mathrm{d}_{4}$, Kynurenic acid- $\mathrm{d}_{5}$, Melatonin- $\mathrm{d}_{4}$, Piconilic acid- $\mathrm{d}_{3}$, Tryptamine- $\mathrm{d}_{4}$, and Xanthurenic acid$\mathrm{d}_{4}$ were purchased from Santa Cruz Biotechnology. Isotopes 3-Hydroxyanthranilic acid- $\mathrm{d}_{4}$, 3Hydroxykynurenine- ${ }^{13} \mathrm{C}_{2}-{ }^{15} \mathrm{~N}$, 5-Hydroxytryptophan- $\mathrm{d}_{4}$, Indole-3-acetamide- $\mathrm{d}_{5}$, Kynurenine$\mathrm{d}_{4}$, and Tryptophan- $\mathrm{d}_{5}$ were purchased from Toronto Research Chemicals. Stock solutions of labeled analytes-were prepared in water with $0.1 \%$ of formic acid. The final concentrations of the internal standards used for analysis are provided in supplementary data (Table A1) and have been chosen to match estimated endogenous metabolite concentrations.

\subsection{Animals}

Adult mice were sacrificed by cervical dislocation and dissected. Urine, blood, liver, cecum and intestinal contents were collected. Samples were individually placed in Eppendorf tubes and kept at $-80^{\circ} \mathrm{C}$ until analysis. All animal experiments were conducted in accordance with local and international guidelines and approved by local Ethics committee.

\subsection{Sample preparations}

\subsubsection{Intestinal contents, cecum and liver tissues}

For better normalization, all samples were freeze-dried (FreeZone ${ }^{\circledR} 4.5$ L, Labconco, USA) at $-107^{\circ} \mathrm{C}, 0.2 \mathrm{mbar}$, for $12 \mathrm{~h}$. Two sample preparation approaches (protocols 1 and 2) were used depending on the sample concentration. Protocol 2 was employed for the extraction of metabolites found at too high concentration to be analyze by LC-HRMS without detector saturation.

Protocol 1: To 3 mg of lyophilized material was added a requisite amount of SIL (see Table A1 of the Supplementary data, for a breakdown of the compositions used) and $900 \mu \mathrm{L}$ of 
$\mathrm{MeOH} / \mathrm{H}_{2} \mathrm{O}$ (1:1). The mixtures were vortexed for $15 \mathrm{~s}$, then homogenized by gentle planar shaking, at $4^{\circ} \mathrm{C}$, for $30 \mathrm{~min}$, followed by centrifugation separation at $15000 \mathrm{xg}$ for $10 \mathrm{~min}$. The supernatant $(900 \mu \mathrm{L})$ was collected and concentrated under vacuum (Savant SPD 111v SpeedVac, Thermo Scientific, USA) at $45^{\circ} \mathrm{C}$ for $4 \mathrm{~h}$. The resultant dry residues were reconstituted in $100 \mu \mathrm{L}$ of $\mathrm{MeOH} / \mathrm{H}_{2} \mathrm{O}$ (1:9) and transferred to a 96-well plate for LC-HRMS analysis.

Protocol 2: Aliquots of Picolinate, QA, Trp, and 3-IS in intestinal contents and cecum all found at higher concentrations, were extracted from $1 \mathrm{mg}$ of lyophilized sample. After addition of SIL internal standards and $500 \mu \mathrm{L}$ of $\mathrm{MeOH} / \mathrm{H}_{2} \mathrm{O}$ (1:9), the samples were vortexed, homogenized and centrifuged as described above. The resultant supernatants were transferred to 96-well plates for LC-HRMS analysis.

\subsubsection{Serum samples}

Protocol 1: Metabolites were extracted from $100 \mu \mathrm{L}$ of serum. After addition of the internal standards (Table A1) and $800 \mu \mathrm{L}$ of $\mathrm{MeOH}$, the samples were vortexed for $15 \mathrm{~s}$ and homogenized at $-20^{\circ} \mathrm{C}$, for $30 \mathrm{~min}$. After centrifugation at $15000 \mathrm{xg}$ for $10 \mathrm{~min}, 800 \mu \mathrm{L}$ of supernatant were collected and concentrated using a SpeedVac. The residues were reconstituted in $100 \mu \mathrm{L}$ of $\mathrm{MeOH} / \mathrm{H}_{2} \mathrm{O}(1: 9)$ and transferred to a 96-well plate for LC-HRMS analysis.

Protocol 2: Higher concentration metabolites (Trp and 3-IS), were extracted from $10 \mu \mathrm{L}$ of serum. After addition of the internal standards and $500 \mu \mathrm{L}$ of $\mathrm{MeOH}$, the samples were vortexed, then homogenized and centrifuged as described above. The supernatant were then directly transferred to a 96-well plate for LC-MS analysis.

\subsubsection{Urine samples}

Metabolites were extracted from $10 \mu \mathrm{L}$ of mice urine. After addition of the internal standards (Table A1) and $240 \mu \mathrm{L}$ of $\mathrm{H}_{2} \mathrm{O}$, the samples were vortexed for $15 \mathrm{~s}$, homogenized at $-20^{\circ} \mathrm{C}$, for 
$30 \mathrm{~min}$ and centrifuged at $15000 \mathrm{xg}$ for $10 \mathrm{~min}$. The supernatant was transferred to a 96 -well plates for LC-HRMS analysis.

\subsection{LC-HRMS analysis}

\subsubsection{Instrumentation}

Analyses were performed as described previously $[14,15]$ (see supplementary data). Briefly, $5 \mu \mathrm{L}$ (or $2 \mu \mathrm{L}$ for protocol 2) were injected into the LC-HRMS system. Chromatography was carried out with a Phenomenex Kinetex $1.7 \mu \mathrm{m} \mathrm{XB} \mathrm{-} \mathrm{C18} \mathrm{(150} \mathrm{mm} \mathrm{x} 2.10 \mathrm{~mm})$ and $100 \AA$ HPLC column maintained at $55^{\circ} \mathrm{C}$. The solvent system comprised mobile phase A $[0.5 \%$ (vol/vol) formic acid in water], and mobile phase B [0.5\% (vol/vol) formic acid in methanol]. Multiple reaction monitoring (MRM) analyses were performed on a UPLC Ultimate WPS-3000 system (Dionex, Germany) coupled to a Q-Exactive mass spectrometer (Thermo Fisher Scientific, Bremen, Germany),that was operated in positive (ESI+) and negative (ESI-) electrospray ionization modes (one run for each mode). Table A2, in supplementary material, listed the exact mass precursor ions, exact mass product ions, retention time (RT), analysis range and normalized collision energy (NCE, \%) for each Trp metabolites.

\subsubsection{Linearity of the method - Limit of quantification (LOQ)}

The calibration curves were obtained by injecting seven standard solutions. The regression parameters of slope, intercept and correlation coefficient were calculated by weight (1/x) linear regression. Linearity was assumed when the regression coefficient was greater than 0.995.

The limit of quantification (LOQ) corresponded to the concentration in the calibration curve producing a signal-to-noise ratio $(\mathrm{S} / \mathrm{N})$ higher than 10 . Signal integration was performed using the filter mode in the Qual Browser window of Xcalibur ${ }^{\circledR}$ software (Thermo Fisher Scientific, San Jose, CA).

\subsubsection{Intra assay accuracy and precision}

Intra-day parameters were evaluated using 10 independent replicates at each level of quality 
control (QC) (a mixture of all injected samples), injected the same day. Three QC levels were used (low, medium and high). The inter-day parameters were evaluated with one independent injection per QC level on 5 separate days. The intra-day and inter-day precisions were restricted to less than $20 \%$ for low level ( $<15 \%$ for higher level), and accuracy was restricted to $\pm 15 \%$ (20\% accepted for the lower level). The accuracy and precision were calculated from results of the analysis of QC samples at high, medium and low concentration levels, both in water and in matrix. Accuracy was defined as the ratio of the difference between the spiked concentration and the observed concentration to the spiked concentration, while precision was defined by the coefficient of variance (CV\%) as the ratio of the standard deviation to the mean.

\subsubsection{Matrix effect}

The matrix effect was evaluated by the comparison of the integration of the analyte at low concentration prepared in water compared with the integration of the spiked analyte at low QC (same concentration) in the studied matrix.

Matrix Effect $(\mathrm{ME} \%)=$ (mean peak area of the spiked analyte in matrix - mean peak area of the endogenous analyte in matrix) / mean peak area of the analyte in water

\subsection{Data processing}

The acquired data were processed using Xcalibur ${ }^{\circledR}$ software (Thermo Fisher Scientific, San Jose, CA) by integrating selected product ion chromatographic peak area, in order to calculate calibration curves and samples concentration.

\subsection{Statistical analysis}

For univariate analysis, statistical analysis was performed using Metaboanalyst, a free web software [16]. 


\section{Results and Discussion}

\subsection{Method development and optimization}

Analyses of blood and urine samples are challenging due to a large sample-to-sample matrix variations and wide ranging basal concentrations of the metabolites involved. Accordingly, multiplex analyses of metabolites for the detection and quantification of individual molecules at differing concentrations is unsurprisingly highly challenging.

Several methods for the analyses of Trp metabolites in plasma and tissue [17, 18], in serum $[19,20]$, in urine $[9,18,20]$, in cerebrospinal fluid $[17,19]$ and in cellular media $[20,21]$ have been described [10]. However, the majority of these methods used a triple quadrupole mass spectrometer. In this study, we have simultaneously quantified Trp and 19 of its metabolites, using an Orbitrap LC-HRMS device (Fig.1). Quantitative analysis was performed by determining the retention times of the precursor ion of each metabolite and at least one fragment ion (Supplementary data, Table A2). The method presented has been validated for 4 different types of matrices by using the internal standard strategy to counteract matrix effects.

Please, insert Fig.1

The study of metabolism in samples from the digestive tract [22, 23] is an emerging field, aimed at the better understanding of microbiota involvement in certain pathologies. These samples require careful pre-analysis, preliminary cleaning of samples of the intestinal contents and tissue extraction of the liver, we chose to lyophilize the samples before metabolite extraction because of their heterogeneity (macromolecules, undigested material, metabolites) [24]. Due to differences in the concentrations of metabolites within the different compartments, we used a dilute approach [18]. This method was employed for metabolites that were present 
at higher concentrations in some matrices as compared to others (Table 1). The total run time of the optimized method was 16.5 min for the simultaneous determination of 20 metabolites.

\section{Please insert Table 1}

\subsection{Method validation}

The presented method was validated for linearity, sensitivity, trueness (accuracy), precision, and matrix effect [25] and it was applied to the quantitation of the considered Trp metabolites in real samples represented by matrices of healthy mice. The linearity of the method was validated from $0.15 \mathrm{nM}$ to $100 \mu \mathrm{M}$ (Table 1 ). The linear correlation coefficient $\left(\mathrm{r}^{2}\right)$ consistently exceeded 0.99, demonstrating satisfactory linearity over two orders of concentration. The limit of quantification (LOQ) ranged from 0.015 to $11.25 \mathrm{nmol} / \mathrm{L}$, with a median value for the 20 metabolites of $0.75 \mathrm{nM}$. The lowest LOQ was obtained for melatonin (LOQ=0.015 nM), and was comparable to those reported in human serum $(0.05 \mathrm{nM})$ [19]. The lowest value of sensitivity was obtained for 5-HIAA with a LOQ of $11.25 \mathrm{nM}$ in good comparable agreement with values obtained by Zhu et al. (between 10 and $50 \mathrm{nM}$ ), using a Triple Quadrupole LC-MS in human urine and serum [20].

For each type of sample, reproducibility, intra- and inter-day trueness were given in Supplementary Data, Tables A3-A6. In the 4 matrices, intra-day precision averaged between 4.9-8.4\%, and intra-day accuracy between 4.6-8.1\% (Table 2). The average precision for all the metabolites also gave a low value of $8.4 \pm 9 \%$, with higher \pm SD compared to the reproducibility. The highest precision was obtained for 3-HAA with reproducibility for the 3 
concentration levels between 2.5 and $6.3 \%$ and accuracy between $-3.6 \%$ and $+4.3 \%$. The lower precision was obtained for ILA with a $\mathrm{CV}=24.3 \%$ at low concentration.

Please, insert Table 2

The matrix effect was determined for each type of sample. Mean recoveries ranged from $62 \%$ to $97 \%$ for the three concentration levels, with the highest difference $( \pm 33 \%$ ) observed in sera (Table 2). These results are consistent with those described previously (86 to 117\%) using a triple quadrupole for urine, plasma and liver [18].

The average risk of ion suppression was highest (mean $34.5 \%$ and $38.4 \%$ for liver and intestinal contents, respectively) for the two solid samples, which had undergone lyophilization followed by solid-liquid extraction. Since recovery was lower than $100 \%$ regardless of the matrix, there was little risk of ionic enhancement due to the matrix and thus, a low risk of false positive results [26].

In the present study, matrix effects could be minimized by dilution at the end of sample preparation. This is one of the reasons we diluted the serum or the intestinal content samples allowing us to quantify the most concentrated metabolites (Table 1). To overcome potential ion suppression, we used internal standards although, previous studies have highlighted the difficulty of quantifying some metabolites, for example, QA was impossible to quantified from mouse brain tissue without deuterated internal standards [17]. In this study, we succeeded in the quantification of QA with a mean recovery in mouse serum of only 20\% (see Supplementary data, Table A3). 


\subsection{Application of the method to mouse samples}

We evaluated the metabolism of Trp in five different types of biological samples for the same mice ( $\mathrm{n}=12$ different animals) i.e. serum, urine, liver, intestinal contents and cecum (this matrix was studied following intestinal contents validation protocol). Metabolite concentrations were matrix dependent as shown in Fig. 2, with a broad range of concentrations. As visualized in Fig. A2 (supplementary material), almost all Trp metabolite concentrations could be determined in the 5 types of matrices. Only 3-hydroxy anthranilate, $N$-acetylserotonin, kynurenate in the liver, and N-AS in the intestinal contents could not be quantified in all replicates, as was melatonin in all matrices. Compared to the analytical variability of our method (Table 2), the inter-individual biological variability proved more important (Supplementary data, Fig. A2). Our results showed that the determination of reference values for these metabolites concentrations is difficult. It was preferable therefore to longitudinally follow the evolution of metabolite concentrations of an individual rather than compare its values to reference values.

Each type of sample had particular sets of tryptophan metabolites. If we consider the intestine which is the most upstream section of the digestive tract, Trp was found in larger quantities. Trp was also the most abundant metabolite in the caecum, but was found at a concentration approximately 7-fold lower than in the intestine.

The high concentration of Trp found in the serum associated with a significant decrease in the concentration along the digestive tract was consistent with a strong intestinal absorption of Trp. These results demonstrated that the absorption of Trp, which is an essential amino acid, was important in the intestine and was concentrated in the serum in order to feed the various metabolic pathways. The second metabolite found at higher concentration in the intestine, caecum and liver was picolinate. This metabolite is implicated in the kynurenine pathway, and indicates that this pathway is particularly active both in the digestive tract and the liver. This is 
further confirmed by the presence in these compartments of kynurenine, and quinolinate but also xanthurenate in the caecum. We also found metabolites of the kynurenine pathway in serum (picolinate) and urine (xanthurenate, kynurenine, quinolinate and picolinate) (see also Supplementary data, Fig. A3). This metabolic pathway can be explored by the quantification of Trp metabolites in each compartment in order to better understand its involvement in inflammatory, immune or neurological processes. Indeed, dysregulation or overstimulation of the kynurenine pathway may lead to activation of the immune system and accumulation of neurotoxic compounds such as quinolinate [27]. Another pathway of Trp metabolism results in the synthesis of serotonin. This monoamine was found in all compartments at fairly high concentrations. The wide variety of actions of serotonin as a neurotransmitter or hormone makes it a messenger and a vital biochemical regulator in vertebrates

The indole derivatives were found in all compartments, but at different concentrations in each type of matrices, suggesting that each of these metabolites may have particular physiological functions (Supplementary data, Fig. A2). Finally, indoxyl sulfate was found at very high concentrations in serum and urine. Moreover, it was the fourth abundant metabolite in the liver and the third abundant in the intestine (Supplementary data, Fig. A3). In contrast, its concentration was low in the caecum relative to other metabolites. These results are consistent with the production of indoxyl sulfate by the liver, from indole derivatives produced by bacteria in the digestive tract. The large quantities of indoxyl sulfate found in most samples make it an important metabolite of the Trp metabolism.

\section{Please, insert Fig.2}

To obtain a global view of the metabolism of Trp at the level of an individual, we carried out a hierarchical grouping study. Fig. 2 shows a heat map representation of this ascending 
hierarchical classification. Samples were grouped according to their similarity in metabolic content. It is interesting to observe that serum and urine fluid samples exhibited Trp metabolite compositions that were different from "solid" samples (cecal and intestinal contents, liver). When serum and urine were compared, the serum had a strong representation of Trp and its metabolites in the associated kynurenine and serotonin (5-HT, N-AS, Kyn, 3-HK) pathways in addition to 3-IS. The different metabolic profiles observed in serum and urine underline the influence of renal function in its role of eliminating potentially toxic metabolites but also of regulation of serum concentrations adapted to normal physiology.

Finally, if we consider the "solid" samples in the hierarchical cluster tree shown in Fig. 2b, liver was separated from cecal and intestinal contents. The liver was characterized by its concentrations of Kyn, Trp and I-3CA. For their parts, intestinal and cecal contents suggest that these regions of the digestive tract have well-defined metabolic roles. It is recognized that circulating serotonin is mainly synthesized in enterochromaffin cells, there were also high concentrations of serotonin from enteric bacterial metabolism. Metabolites of the Kynurenine pathway (XA, 3-HAA, QA, Picolinate, and KA) as well as the indole pathway (both involved in inflammatory phenomena) were characteristic of the caecum.

\section{CONCLUSIONS}

The metabolism of Trp generates a large number of metabolites whose physiological roles are important and diverse. For this purpose, we have developed and validated a sensitive and robust analytical approach, based on LC-HRMS, which makes it possible to quantify the concentrations of Trp metabolites in five different types of samples. This has the potential for not only identification of pathological dysfunctions, but also to follow the evolution of metabolites in response to therapeutic action. 


\section{ASSOCIATED CONTENT}

\section{Supporting Information}

Supporting Information contains description of material and methods; Fig. A1. Structures of the 20 tryptophan metabolites quantified in this study; Table A1.Stable isotope labelled internal standards and their concentration for sample preparation; Table A2. Metabolites, and corresponding standards, and their identification; Tables A3-A6. Precision, accuracy and matrix effect at low, medium and high QC levels in mouse serum, urines, cecum, respectively; Fig. A2. Mouse metabolite concentrations in various matrices and Fig. A3. Rank -Higher to lower concentration intensity of a metabolite in mice matrix.

\section{ACKNOWLEDGEMENTS}

This work was supported by the "Institut National de la Santé et de la Recherche” INSERM and the University of Tours. We thank the department “Analyse des Systèmes Biologiques” (PST ASB, Université de Tours, France) for their help with sample analyses. H.S. received funding from the European Research Council (ERC) under the European Union’s Horizon 2020 Research and Innovation Programme (ERC-2016-StG-71577).

\section{REFERENCES}

[1] D. Keszthelyi, F.J. Troost, A.A. Masclee, Understanding the role of tryptophan and serotonin metabolism in gastrointestinal function, Neurogastroenterol. Motil. 21(12) (2009) 1239-49.

[2] L.M. Alkhalaf, K.S. Ryan, Biosynthetic manipulation of tryptophan in bacteria: pathways and mechanisms, Chem. Biol. 22(3) (2015) 317-28. 
[3] P.J. Kennedy, J.F. Cryan, T.G. Dinan, G. Clarke, Kynurenine pathway metabolism and the microbiota-gut-brain axis, Neuropharmacology 112(Part B) (2017) 399-412.

[4] G. Clarke, D.P. McKernan, G. Gaszner, E.M. Quigley, J.F. Cryan, T.G. Dinan, A Distinct Profile of Tryptophan Metabolism along the Kynurenine Pathway Downstream of Toll-Like Receptor Activation in Irritable Bowel Syndrome, Front. Pharmacol. 3 (2012) 90.

[5] G.M. Mawe, J.M. Hoffman, Serotonin signalling in the gut--functions, dysfunctions and therapeutic targets, Nat. Rev. Gastroenterol. Hepatol. 10(8) (2013) 473-86.

[6] E.E. Alexeev, J.M. Lanis, D.J. Kao, E.L. Campbell, C.J. Kelly, K.D. Battista, M.E. Gerich, B.R. Jenkins, S.T. Walk, D.J. Kominsky, S.P. Colgan, Microbiota-Derived Indole Metabolites Promote Human and Murine Intestinal Homeostasis through Regulation of Interleukin-10 Receptor, Am. J. Pathol. 188(5) (2018) 1183-94.

[7] T.D. Hubbard, I.A. Murray, G.H. Perdew, Indole and Tryptophan Metabolism: Endogenous and Dietary Routes to Ah Receptor Activation, Drug Metab. Dispos. 43(10) (2015) 1522-35.

[8] A. Agus, J. Planchais, H. Sokol, Gut Microbiota Regulation of Tryptophan Metabolism in Health and Disease, Cell Host Microbe 23(6) (2018) 716-24.

[9] J.S. Oh, H.S. Seo, K.H. Kim, H. Pyo, B.C. Chung, J. Lee, Urinary profiling of tryptophan and its related metabolites in patients with metabolic syndrome by liquid chromatographyelectrospray ionization/mass spectrometry, Anal. Bioanal. Chem. 409(23) (2017) 5501-12.

[10] I. Sadok, A. Gamian, M.M. Staniszewska, Chromatographic analysis of tryptophan metabolites, J. Sep. Sci. 40(15) (2017) 3020-45.

[11] X. Zheng, A. Kang, C. Dai, Y. Liang, T. Xie, L. Xie, Y. Peng, G. Wang, H. Hao, Quantitative analysis of neurochemical panel in rat brain and plasma by liquid chromatography-tandem mass spectrometry, Anal. Chem. 84(22) (2012) 10044-51.

[12] K. Arnhard, F. Pitterl, B. Sperner-Unterweger, D. Fuchs, T. Koal, H. Oberacher, A validated liquid chromatography-high resolution-tandem mass spectrometry method for the simultaneous quantitation of tryptophan, kynurenine, kynurenic acid, and quinolinic acid in human plasma, Electrophoresis 39(9-10) (2018) 1171-80.

[13] Q. Tong, J. Song, G.J. Yang, L. Fan, W. Xiong, J.G. Fang, Simultaneous determination of tryptophan, kynurenine, kynurenic acid, xanthurenic acid and 5-hydroxytryptamine in human plasma by LC-MS/MS and its application to acute myocardial infarction monitoring, Biomed. Chromatogr. 32(4) (2018).

[14] B. Diémé, A. Lefèvre, L. Nadal-Desbarats, L. Galineau, B. Madji Hounoum, F. Montigny, H. Blasco, C.R. Andres, P. Emond, S. Mavel, Workflow methodology for rat brain metabolome exploration using NMR, LC-MS and GC-MS analytical platforms, J. Pharm. Biomed. Anal. 142 (2017) 270-8.

[15] B. Madji Hounoum, H. Blasco, L. Nadal-Desbarats, B. Diémé, F. Montigny, C.R. Andres, P. Emond, S. Mavel, Analytical methodology for metabolomics study of adherent mammalian cells using NMR, GC-MS and LC-HRMS, Anal. Bioanal. Chem. 407(29) (2015) 8861-72.

[16] J. Xia, D.S. Wishart, Using MetaboAnalyst 3.0 for Comprehensive Metabolomics Data Analysis, Curr. Protoc. Bioinformatics 55 (2016) 14.10.1-14.10.91.

[17] R. Fuertig, A. Ceci, S.M. Camus, E. Bezard, A.H. Luippold, B. Hengerer, LC-MS/MS-based quantification of kynurenine metabolites, tryptophan, monoamines and neopterin in plasma, cerebrospinal fluid and brain, Bioanalysis 8(18) (2016) 1903-17.

[18] J. Marcos, N. Renau, O. Valverde, G. Aznar-Lain, I. Gracia-Rubio, M. Gonzalez-Sepulveda, L.A. Perez-Jurado, R. Ventura, J. Segura, O.J. Pozo, Targeting tryptophan and tyrosine metabolism by liquid chromatography tandem mass spectrometry, J. Chromatogr. A 1434 (2016) 91-101. 
[19] E. Henykova, H.P. Vranova, P. Amakorova, T. Pospisil, A. Zukauskaite, M. Vlckova, L. Urbanek, O. Novak, J. Mares, P. Kanovsky, M. Strnad, Stable isotope dilution ultra-high performance liquid chromatography-tandem mass spectrometry quantitative profiling of tryptophan-related neuroactive substances in human serum and cerebrospinal fluid, J. Chromatogr. A 1437 (2016) 145-57.

[20] W. Zhu, A.P. Stevens, K. Dettmer, E. Gottfried, S. Hoves, M. Kreutz, E. Holler, A.B. Canelas, I. Kema, P.J. Oefner, Quantitative profiling of tryptophan metabolites in serum, urine, and cell culture supernatants by liquid chromatography-tandem mass spectrometry, Anal. Bioanal. Chem. 401(10) (2011) 3249-61.

[21] E. Prinsen, W. VanDongen, E.L. Esmans, H.A. VanOnckelen, HPLC linked electrospray tandem mass spectrometry: A rapid and reliable method to analyse indole-3-acetic acid metabolism in bacteria, J. Mass Spectrom. 32(1) (1997) 12-22.

[22] T.J. Anderson, R.W. Jones, Y. Ai, R.S. Houk, J.-I. Jane, Y. Zhao, D.F. Birt, J.F. McClelland, High-resolution time-of-flight mass spectrometry fingerprinting of metabolites from cecum and distal colon contents of rats fed resistant starch, Anal. Bioanal. Chem. 406(3) (2014) 74556.

[23] Z. Yang, J.-J. Hou, P. Qi, M. Yang, B.-P. Yan, Q.-R. Bi, R.-H. Feng, W.-Z. Yang, W.-Y. Wu, D.A. Guo, Colon-derived uremic biomarkers induced by the acute toxicity of Kansui radix: $A$ metabolomics study of rat plasma and intestinal contents by UPLC-QTOF-MSE, J. Chromatogr. B 1026 (2016) 193-203.

[24] O. Deda, H.G. Gika, I.D. Wilson, G.A. Theodoridis, An overview of fecal sample preparation for global metabolic profiling, J. Pharm. Biomed. Anal. 113 (2015) 137-50.

[25] The International Council for Harmonisation (ICH), ICH Quality Guidelines ICH Quality Guidelines: An Implementation Guide "Q2(R1) Validation of Analytical Procedures: Text and Methodology", John Wiley \& Sons, Inc., Hoboken, N. J. 2018.

[26] A. Furey, M. Moriarty, V. Bane, B. Kinsella, M. Lehane, lon suppression; A critical review on causes, evaluation, prevention and applications, Talanta 115(Supplement C) (2013) 10422.

[27] R. Schwarcz, J.P. Bruno, P.J. Muchowski, H.Q. Wu, Kynurenines in the mammalian brain: when physiology meets pathology, Nat. Rev. Neurosci. 13(7) (2012) 465-77. 


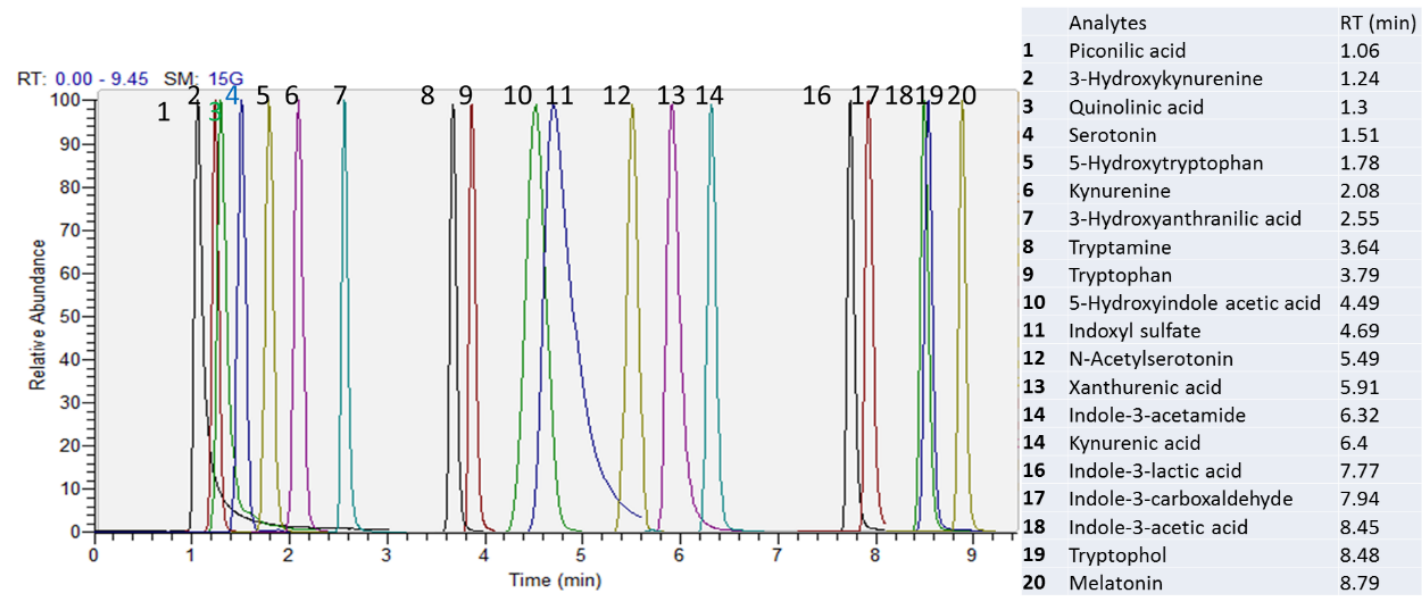

Fig. 1. Extracted ion chromatogram of the compilation of 20 Trp catabolites 


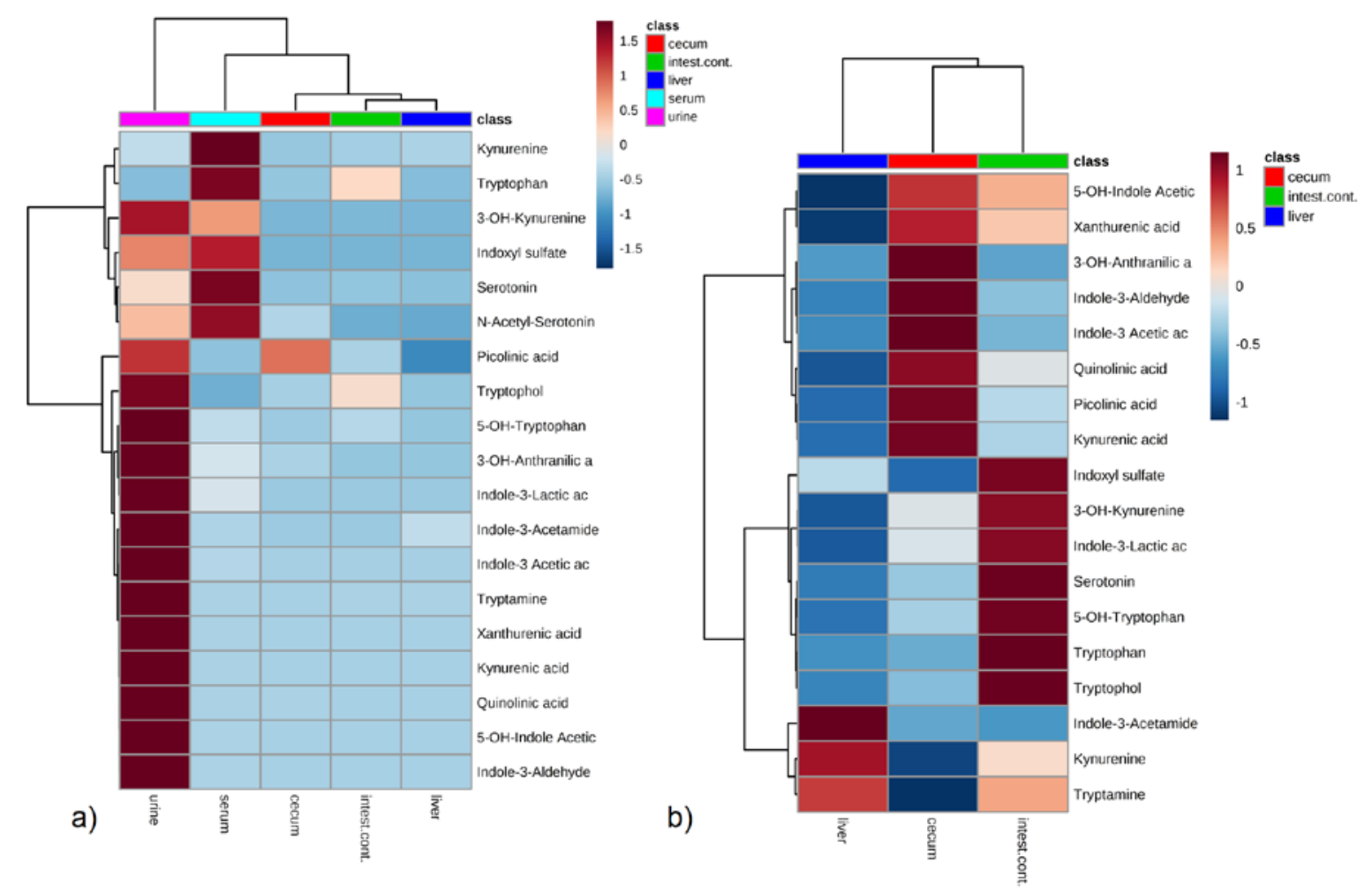

Fig. 2. Hierarchical clustering shown as heatmap visualizing the correlation between metabolites and matrices (distance measure using Euclidean, and clustering algorithm using Ward.D) (a) in the 5 matrices; (b) in solid matrices. 
Table 1. Range of linearity (correlation coefficient $r^{2}>0.99$ ) for the 20 metabolites in the different matrices and limit of quantification (LOQ).

\begin{tabular}{|c|c|c|c|c|c|c|}
\hline \multirow[b]{2}{*}{ Metabol. } & \multirow[b]{2}{*}{$\begin{array}{l}\text { Internal } \\
\text { Standard }\end{array}$} & \multicolumn{4}{|c|}{ Dynamic range (nmol/L) } & \multirow[b]{2}{*}{$\begin{array}{l}\mathrm{LOQ} \\
(\mathrm{nmol} / \mathrm{L})\end{array}$} \\
\hline & & Serum & $\begin{array}{l}\text { Intestinal } \\
\text { cont. }\end{array}$ & Liver & Urine & \\
\hline 3-HAA & 3-OH-AA-d 4 & $1.5-1000$ & $1.5-1000$ & $3.75-2500$ & $7.5-5000$ & 0.1 \\
\hline 3-HK & $\begin{array}{l}\text { 3-OH-KYN- } \\
{ }^{13} \mathrm{C}_{2}-{ }^{15} \mathrm{~N}\end{array}$ & $1.5-1000$ & $0.75-500$ & $0.75-500$ & $7.5-1500$ & 1.5 \\
\hline 3-IS & $\mathrm{KA}-\mathrm{d}_{5}$ & $112.5-75000$ & 7.5-5000* & 7.5-5000 & $125-75000$ & 7.5 \\
\hline 5-HIAA & 5-HIAA-d ${ }_{5}$ & $30-20000$ & $30-20000$ & $3.75-2500$ & $125-25000$ & 11.25 \\
\hline 5-НРТ & 5-OH-TRP-d 4 & $2.25-1500$ & $1.5-1000$ & $0.75-500$ & $3.75-2500$ & 1.125 \\
\hline 5-HT & $5-\mathrm{HT}-\mathrm{d}_{4}$ & $150-100000^{*}$ & $11.25-7500$ & $22.5-15000$ & $10-2000$ & 1.125 \\
\hline I-3CA & I-3-AA- $\mathrm{d}_{4}$ & $7.5-5000$ & $11.25-7500$ & $0.75-500$ & $2.5-500$ & 0.75 \\
\hline IAA & I-3-AA- $d_{4}$ & $37.5-25000^{*}$ & $3.75-2500$ & $0.75-500$ & $5-100$ & 0.75 \\
\hline IAM & $\mathrm{I}-3-\mathrm{AcNH}_{2}-\mathrm{d}_{5}$ & $0.15-100$ & $0.15-100$ & $0.15-100$ & $2.5-500$ & 0.15 \\
\hline ILA & $\mathrm{XA}-\mathrm{d}_{4}$ & $75-50000 *$ & $11.25-7500$ & $0.75-500$ & $125-2500$ & 0.75 \\
\hline KA & $\mathrm{KYN}-\mathrm{d}_{4}$ & $75-50000$ & $3.75-2500$ & $15-10000$ & $125-25000$ & 0.75 \\
\hline Kyn & $\mathrm{KA}-\mathrm{d}_{5}$ & $11.25-7500 *$ & $4.5-3000$ & $2.25-1500$ & $150-1500$ & 0.75 \\
\hline Melatonin & ${\mathrm{MEL}-\mathrm{d}_{4}}_{4}$ & $0.15-100$ & $0.15-100$ & $0.15-100$ & $0.5-100$ & 0.015 \\
\hline N-AS & XA-d 4 & $0.75-500$ & $0.75-500$ & $0.75-500$ & $2.5-500$ & 0.75 \\
\hline Piconilate & $\mathrm{PICO}-\mathrm{d}_{3}$ & $150-100000 *$ & $15-10000 *$ & $15-10000$ & $50-5000$ & 3.75 \\
\hline QA & $\mathrm{PICO}-\mathrm{d}_{3}$ & $1.13-750$ & $2.25-1500$ * & $15-10000$ & $150-1500$ & 1.125 \\
\hline TA & $\begin{array}{l}\text { Tryptamine- } \\
\mathrm{d}_{4}\end{array}$ & $0.75-500$ & $2.25-1500$ & $0.75-500$ & $7.5-5000$ & 0.25 \\
\hline Trp & TRP-d ${ }_{5}$ & $150-100000 *$ & $75-50000 *$ & $75-50000$ & $0.125-25$ & 3 \\
\hline Tryptophol & $\mathrm{KA}-\mathrm{d}_{5}$ & $0.38-250$ & $3.75-2500$ & $0.75-500$ & $22.5-1500$ & 0.375 \\
\hline XA & $\mathrm{XA}-\mathrm{d}_{4}$ & $3-2000$ & $3-2000$ & $0.75-500$ & $125-75000$ & 0.75 \\
\hline
\end{tabular}

* Molecules serially diluted due to higher concentration in the matrix 
Table 2. Mean values of 20 metabolites for precision, accuracy (in absolute terms) and matrix effect in the 4 matrices

\begin{tabular}{|l|l|l|l|l|l|}
\hline \multirow{2}{*}{ Matrix } & \multicolumn{2}{|l|}{ Intra-day $(\mathrm{n}=10)$} & \multicolumn{2}{|l|}{ Inter-day $(\mathrm{n}=5)$} & $\begin{array}{l}\text { Matrix effect } \\
(\mathrm{n}=10)\end{array}$ \\
\cline { 2 - 6 } & CV (\%) & $\begin{array}{l}\text { Accuracy } \\
(\%)\end{array}$ & CV (\%) $\begin{array}{r}\text { Accuracy } \\
(\%)\end{array}$ & Recovery (\%) \pm sd \\
\hline Serum & $4.6 \pm 2$ & $5.5 \pm 4$ & $7.2 \pm 5$ & $5.0 \pm 3$ & $80.1 \pm 33$ \\
\hline Liver & $7.0 \pm 5$ & $8.4 \pm 9$ & $8.5 \pm 4.9$ & $4.9 \pm 4.0$ & $65.5 \pm 24$ \\
\hline Intest. cont. & $8.1 \pm 5$ & $7.5 \pm 5$ & $8.9 \pm 8.5$ & $6.3 \pm 6$ & $61.6 \pm 28$ \\
\hline Urine & $5.0 \pm 2.7$ & $4.9 \pm 4.4$ & $6.6 \pm 4.0$ & $3.5 \pm 2.4$ & $97.1 \pm 15$ \\
\hline
\end{tabular}




\section{APPENDICES}

\section{SUPPLEMENTARY DATA}

\section{Validation of global quantitative analysis methodology of tryptophan metabolites in mice using LC-MS}

Antoine Lefèvre ${ }^{a}$, Sylvie Mavel ${ }^{a}$, Lydie Nadal-Desbarats ${ }^{a}$, Laurent Galineau ${ }^{a}$, Sylvie Attucci ${ }^{a}$, Diane Dufour $^{\mathrm{a}, \mathrm{b}}$, Harry Sokol ${ }^{\mathrm{c}, \mathrm{d}}$, Patrick Emond ${ }^{\mathrm{a}, \mathrm{b}^{*}}$

\section{Contents :}

\section{LC-HRMS experiment, Instrumentation}

Table A1. Stable isotope labelled internal standards and their concentration for sample preparation.

Table A2. Metabolites, and corresponding standards, and their identification.

Table A3. Precision, accuracy and matrix effect at low, medium and high QC levels in mouse serum

Table A4. Precision, accuracy and matrix effect at low, medium and high QC levels in mouse urines

Table A5. Precision, accuracy and matrix effect at low, medium and high QC levels in mouse cecum 


\section{Complementary data for LC-HRMS instrumentation}

The UHPLC autosampler temperature was set at $4^{\circ} \mathrm{C}$ and the injection volume for each sample was 5 $\mu \mathrm{L}$. The HESI (heated electrospray ionization) source was used for both modes, with a spray voltage of 4.5 kV, a capillary temperature of $250{ }^{\circ} \mathrm{C}$, a heater temperature of $475^{\circ} \mathrm{C}$, a sheath gas flow of 35 arbitrary units (AU), an auxiliary gas flow of $10 \mathrm{AU}$, a spare gas flow of $1 \mathrm{AU}$, and a tube lens voltage of 100 V. During the Parallel Reaction monitoring (PRM) acquisition, the instrument operated at 35,000 resolution $(\mathrm{m} / \mathrm{z}=200)$, with an automatic gain control (AGC) target of $2 \times 10^{5}$ charges and a maximum injection time (IT) of $50 \mathrm{~ms}$. Instrumental chromatography stability was evaluated by multiple injections $(x=15)$ of a quality control $(Q C)$ sample obtained from a pool of $10 \mu \mathrm{L}$ of all samples analyzed. This QC sample was reinjected once at the beginning of the analysis, every 10 sample injections, and at the end of the run. 
Table A1. Stable isotope labelled internal standards and their concentration (in nmol/L) as used in sample preparation.

\begin{tabular}{|c|c|c|c|c|c|}
\hline & & Serum & $\begin{array}{l}\text { Cecum } \\
\text { Intest. } \\
\text { Contents }\end{array}$ & Liver & Urine \\
\hline 5-Hydroxyindole acetic acid- $\mathrm{d}_{5}$ & 5-HIAA-d 5 & 750 & 1000 & 100 & 1000 \\
\hline Serotonin- $\mathrm{d}_{4}$ & $5-\mathrm{HT}-\mathrm{d}_{4}$ & 1000 & 750 & 750 & 250 \\
\hline Indole-3-acetic acid- $\mathrm{d}_{4}$ & I-3-AA-d 4 & 1000 & 300 & 200 & 500 \\
\hline Kynurenic acid- $\mathrm{d}_{5}$ & KA-d ${ }_{5}$ & 500 & 200 & 200 & 1000 \\
\hline Melatonin- $\mathrm{d}_{4}$ & $\mathrm{MEL} \mathrm{d}_{4}$ & 20 & 20 & 20 & 20 \\
\hline Piconilic acid-d 3 & ${\mathrm{PICO}-\mathrm{d}_{3}}$ & 2000 & 500 & 750 & 750 \\
\hline Tryptamine- $\mathrm{d}_{4}$ & Tryptamine-d 4 & 250 & 50 & 100 & 200 \\
\hline Xanthurenic acid- $\mathrm{d}_{4}$ & $\mathrm{XA}-\mathrm{d}_{4}$ & 500 & 100 & 200 & 1000 \\
\hline 3-Hydroxyanthranilic acid-d 4 & $3-\mathrm{OH}-\mathrm{AA}-\mathrm{d}_{4}$ & 500 & 500 & 500 & 1000 \\
\hline 3-Hydroxykynurenine- ${ }^{13} \mathrm{C}_{2}-{ }^{15} \mathrm{~N}$ & $3-\mathrm{OH}-\mathrm{KYN}-{ }^{13} \mathrm{C}_{2}-{ }^{15} \mathrm{~N}$ & 200 & 300 & 200 & 100 \\
\hline 5-Hydroxytryptophan- $\mathrm{d}_{4}$ & 5-OH-TRP- $\mathrm{d}_{4}$ & 200 & 500 & 200 & 200 \\
\hline Indole-3-acetamide- $\mathrm{d}_{5}$ & I-3-AcNH2-d 5 & 200 & 25 & 100 & 100 \\
\hline Kynurenine- $\mathrm{d}_{4}$ & $\mathrm{KYN}^{-\mathrm{d}_{4}}$ & 750 & 500 & 500 & 100 \\
\hline Tryptophan- $\mathrm{d}_{5}$ & TRP-d 5 & 10000 & 2000 & 2000 & 1000 \\
\hline
\end{tabular}


Table A2. Metabolites, and corresponding standards, and their identification. The underlined precursor ion was used as the quantifying ion.

\begin{tabular}{|c|c|c|c|c|c|c|}
\hline Analytes & Abbreviation & \begin{tabular}{|l} 
Precursor \\
ion $(m / z)$ \\
{$[\mathrm{M}+\mathrm{H}]$} \\
\end{tabular} & $\begin{array}{l}\mathrm{RT} \\
(\min )\end{array}$ & $\begin{array}{l}\text { Analysis } \\
\text { Range } \\
\text { (min) }\end{array}$ & $\begin{array}{l}\text { NCE } \\
(\%)\end{array}$ & Products Ion $(\mathrm{m} / \mathrm{z})$ \\
\hline 3-Hydroxyanthranilic acid & 3-HAA & 154.04987 & 2.6 & $2.4-3.2$ & 15 & $\underline{136.03917}$ \\
\hline $\begin{array}{l}\text { 3-Hydroxyanthranilic acid- } \\
\mathrm{d}_{4}\end{array}$ & 3-OH-AA-d 4 & 157.06869 & 2.6 & $2.4-3.2$ & 15 & $\underline{139.05791}$ \\
\hline 3-Hydroxykynurenine & 3-HK & 225.08698 & 1.2 & $0-1.6$ & 25 & $\begin{array}{l}\text { 110.06016; 162.05458; } \\
208.05997\end{array}$ \\
\hline $\begin{array}{l}\text { 3-Hydroxykynurenine- } \\
{ }^{13} \mathrm{C}_{2}-{ }^{15} \mathrm{~N}\end{array}$ & $\begin{array}{l}3-\mathrm{OH}-\mathrm{KYN}- \\
{ }^{13} \mathrm{C}_{2}-{ }^{15} \mathrm{~N}\end{array}$ & 228.09072 & 1.2 & $0-1.6$ & 25 & $\begin{array}{l}\text { 110.06036; 163.05821; } \\
210.06660\end{array}$ \\
\hline $\begin{array}{l}\text { 5-Hydroxyindole acetic } \\
\text { acid }\end{array}$ & 5-HIAA & 192.06552 & 4.6 & $4.1-5.4$ & 45 & $\underline{146.05974}$ \\
\hline $\begin{array}{l}\text { 5-Hydroxyindole acetic } \\
\text { acid- } \mathrm{d}_{5}\end{array}$ & $5-\mathrm{HIAA} \mathrm{d}_{5}$ & 196.08907 & 4.6 & $4.1-5.4$ & 45 & 150.08482 \\
\hline 5-Hydroxytryptophan & 5-HPT & 221.09207 & 1.8 & $1.4-2.4$ & 45 & $162.05457 ; 204.06511$ \\
\hline 5-Hydroxytryptophan-d ${ }_{4}$ & 5-OH-TRP- $\mathrm{d}_{4}$ & 225.11717 & 1.8 & $1.4-2.4$ & 45 & $165.07362 ; 207.08412$ \\
\hline Indole-3-acetamide & IAM & 175.08659 & 6.4 & $5.9-7.3$ & 55 & 130.06500 \\
\hline Indole-3-acetamide- $d_{5}$ & $\begin{array}{l}\text { I-3-AcNH2- } \\
d_{5}\end{array}$ & 180.11797 & 6.4 & $5.9-7.3$ & 55 & $\underline{134.09003}$ \\
\hline Indole-3-acetic acid & IAA & 176.07061 & 8.6 & $8.3-9.45$ & 55 & 130.06505 \\
\hline Indole-3-acetic acid- $\mathrm{d}_{4}$ & I-3-AA- $d_{4}$ & 180.09571 & 8.6 & $8.3-9.45$ & 55 & 133.08383 \\
\hline Indole-3-carboxaldehyde & I-3CA & 146.06004 & 8.1 & $7.3-8.3$ & 65 & $91.05459 ; \underline{118.06518}$ \\
\hline Indole-3-lactic acid & ILA & 206.08116 & 7.9 & $7.3-8.3$ & 65 & $\frac{118.06510}{170.05966} ; 130.06502 ;$ \\
\hline Indoxyl sulfate & $3-I S$ & 212.00145 & 4.95 & $4.1-5.4$ & 75 & 79.95586; 132.04425 \\
\hline Kynurenic acid & $\mathrm{KA}$ & 190.04987 & 6.5 & $5.9-7.3$ & 55 & 162.05458 \\
\hline Kynurenic acid- $d_{5}$ & $K A-d_{5}$ & 195.08125 & 6.5 & $5.9-7.3$ & 55 & 167.08594 \\
\hline Kynurenine & Kyn & 209.09207 & 2.1 & $1.6-2.4$ & 25 & $\begin{array}{l}\text { 94.065450; 136.07552; } \\
192.06512 \\
\end{array}$ \\
\hline Kynurenine- $d_{4}$ & $\mathrm{KYN}-\mathrm{d}_{4}$ & 213.11717 & 2.1 & $1.6-2.4$ & 25 & $\begin{array}{l}\text { 98.09051; 140.10054; } \\
196.09024 \\
\end{array}$ \\
\hline Melatonin & & 233.12845 & 8.9 & $8.3-9.45$ & 35 & $\underline{174.09096}$ \\
\hline Melatonin- $\mathrm{d}_{4}$ & MEL-d ${ }_{4}$ & 237.15356 & 8.9 & $8.3-9.45$ & 35 & $\underline{178.11606}$ \\
\hline N-Acetylserotonin & N-AS & 219.11280 & 5.6 & $5.4-5.9$ & 35 & $\underline{160.07530}$ \\
\hline Piconilic acid & & 124.03931 & 1 & $0-3.5$ & 85 & 78.034440; 96.04482 \\
\hline Piconilic acid- $d_{3}$ & $\mathrm{PICO}-\mathrm{d}_{3}$ & 127.05813 & 1 & $0-3.5$ & 85 & 80.047180; 99.06388 \\
\hline Quinolinic acid & QA & 168.02913 & 1.25 & $0-3.5$ & 75 & 96.044930 \\
\hline Serotonin & $5-\mathrm{HT}$ & 177.10224 & 1.5 & $0-2.4$ & 45 & $132.08070 ; 160.07539$ \\
\hline Serotonin- $d_{4}$ & $5-\mathrm{HT}-\mathrm{d}_{4}$ & 181.12734 & 1.5 & $0-2.4$ & 45 & $136.10570 ; 164.10049$ \\
\hline Tryptamine & TA & 161.10733 & 3.6 & $3.2-4.1$ & 45 & 144.08051 \\
\hline
\end{tabular}




\begin{tabular}{|l|l|l|l|l|l|l|}
\hline Tryptamine- $\mathrm{d}_{4}$ & $\begin{array}{l}\text { Tryptamine- } \\
\mathrm{d}_{4}\end{array}$ & 165.13243 & 3.6 & $3.2-4.1$ & 45 & $\underline{148.10551}$ \\
\hline Tryptophan & Trp & 205.09715 & 3.9 & $3.2-4.1$ & 15 & $146.05988 . \underline{188.07039}$ \\
\hline Tryptophan- $\mathrm{d}_{5}$ & TRP- $\mathrm{d}_{5}$ & 210.12853 & 3.9 & $3.2-4.1$ & 15 & $150.08511 ; \underline{192.09557}$ \\
\hline Tryptophol & & 162.09134 & 8.6 & $8.3-9.45$ & 45 & $\underline{144.08054}$ \\
\hline Xanthurenic acid & XA & 206.04478 & 6 & $5.9-7.3$ & 55 & $132.04417 ; \underline{178.04941}$ \\
\hline Xanthurenic acid- $\mathrm{d}_{4}$ & XA- $\mathrm{d}_{4}$ & 210.06989 & 6 & $5.9-7.3$ & 55 & $136.06935 ; \underline{182.07469}$ \\
\hline
\end{tabular}


Table A3. Precision, accuracy and matrix effect at low, medium and high QC levels in mouse serum

\begin{tabular}{|c|c|c|c|c|c|c|}
\hline \multirow{2}{*}{ Metabolites } & \multirow{2}{*}{$\begin{array}{l}\text { Concentration } \\
\qquad(\mathrm{nmol} / \mathrm{L})\end{array}$} & \multicolumn{2}{|c|}{ Intra-day $(n=10)$} & \multicolumn{2}{|c|}{ Inter-day $(n=5)$} & \multirow{2}{*}{$\begin{array}{l}\text { Matrix effect } \\
(n=10)\end{array}$} \\
\hline & & $\begin{array}{l}\mathrm{CV} \\
(\%)\end{array}$ & $\begin{array}{c}\text { Accuracy } \\
\text { (\%) }\end{array}$ & $\begin{array}{l}\mathrm{CV} \\
(\%)\end{array}$ & $\begin{array}{c}\text { Accuracy } \\
\text { (\%) }\end{array}$ & \\
\hline \multirow[t]{3}{*}{ IAA* } & 1 & 5.54 & 7.98 & 3.68 & 10.40 & $78 \pm 8$ \\
\hline & 10 & 5.70 & -0.89 & 11.18 & -5.45 & $94 \pm 8$ \\
\hline & 20 & 1.51 & -7.41 & 16.97 & -12.30 & $111 \pm 6$ \\
\hline \multirow{3}{*}{ ILA* } & 2 & 3.61 & 8.86 & 2.52 & 8.43 & $88 \pm 6$ \\
\hline & 20 & 5.27 & 3.16 & 5.78 & 1.25 & $105 \pm 4$ \\
\hline & 40 & 2.14 & -2.14 & 11.87 & -3.14 & $119 \pm 4$ \\
\hline \multirow[t]{3}{*}{ Kyn* } & 2 & 2.16 & 8.71 & 2.70 & 10.61 & $60 \pm 3$ \\
\hline & 20 & 3.19 & 4.65 & 3.26 & 7.17 & $58 \pm 4$ \\
\hline & 40 & 6.90 & 1.38 & 3.78 & 6.25 & $63 \pm 4$ \\
\hline \multirow[t]{3}{*}{$3-I S^{*}$} & 3 & 9.28 & 1.26 & 20.58 & 1.33 & $113 \pm 13$ \\
\hline & 30 & 3.20 & 4.42 & 17.47 & 0.16 & $112 \pm 3$ \\
\hline & 60 & 8.43 & -0.11 & 1.29 & 6.90 & $119 \pm 11$ \\
\hline \multirow[t]{3}{*}{$5-\mathrm{HT}^{*}$} & 4 & 3.53 & 7.08 & 4.56 & 8.14 & $66 \pm 4$ \\
\hline & 40 & 4.49 & 3.77 & 3.31 & 5.16 & $56 \pm 4$ \\
\hline & 80 & 4.35 & 3.92 & 4.52 & 3.16 & $54 \pm 3$ \\
\hline \multirow[t]{3}{*}{ IAM } & 4 & 4.09 & 14.96 & 7.23 & -1.13 & $77 \pm 32$ \\
\hline & 40 & 9.13 & -0.35 & 10.12 & -5.93 & $78 \pm 28$ \\
\hline & 80 & 5.05 & -7.62 & 10.41 & 12.56 & $69 \pm 27$ \\
\hline \multirow[t]{3}{*}{ Melatonin } & 4 & 2.80 & 6.44 & 5.95 & 6.55 & $103 \pm 6$ \\
\hline & 40 & 2.15 & -3.82 & 2.96 & -0.01 & $102 \pm 10$ \\
\hline & 80 & 2.57 & -2.09 & 1.71 & -0.18 & $117 \pm 6$ \\
\hline \multirow[t]{3}{*}{ Piconilic acid* } & 4 & 3.45 & 1.62 & 2.59 & 1.47 & $25 \pm 2$ \\
\hline & 40 & 2.16 & -5.87 & 9.35 & -1.21 & $23 \pm 1$ \\
\hline & 80 & 3.83 & -2.35 & 5.54 & 1.83 & $27 \pm 1$ \\
\hline \multirow[t]{3}{*}{ Trp* } & 4 & 2.97 & 9.59 & 1.69 & 11.86 & $115 \pm 10$ \\
\hline & 40 & 3.08 & 0.01 & 3.91 & 0.33 & $114 \pm 3$ \\
\hline & 80 & 4.32 & -5.20 & 1.52 & -9.07 & $115 \pm 6$ \\
\hline \multirow[t]{3}{*}{ Tryptophol } & 10 & 4.01 & 8.04 & 3.62 & 9.90 & $87 \pm 7$ \\
\hline & 100 & 6.23 & -0.24 & 6.92 & -1.17 & $94 \pm 6$ \\
\hline & 200 & 4.68 & -3.42 & 3.74 & -6.17 & $111 \pm 8$ \\
\hline \multirow[t]{3}{*}{ N-AS } & 20 & 8.84 & -7.14 & 11.07 & -6.82 & $98 \pm 9$ \\
\hline & 200 & 3.05 & -12.75 & 12.02 & -6.96 & $104 \pm 7$ \\
\hline & 400 & 10.45 & -4.38 & 11.70 & -5.51 & $110 \pm 11$ \\
\hline \multirow[t]{3}{*}{ TA } & 20 & 2.48 & 0.44 & 2.72 & 3.43 & $98 \pm 5$ \\
\hline & 200 & 4.25 & -4.51 & 6.92 & 2.60 & $93 \pm 7$ \\
\hline & 400 & 4.57 & -5.49 & 4.33 & -1.84 & $101 \pm 5$ \\
\hline \multirow[t]{3}{*}{ QA } & 30 & 5.35 & 6.35 & 17.44 & -2.10 & $12 \pm 1$ \\
\hline & 300 & 5.66 & -8.84 & 15.00 & -4.65 & $22 \pm 1$ \\
\hline & 600 & 3.67 & -2.52 & 11.68 & 5.17 & $24 \pm 1$ \\
\hline
\end{tabular}




\begin{tabular}{|l|c|cc|cc|c|}
\hline 3-HAA & 40 & 5.32 & -3.70 & 10.06 & -5.97 & $80 \pm 6$ \\
& 400 & 5.71 & -13.38 & 7.38 & 1.05 & $76 \pm 4$ \\
& 800 & 4.29 & -14.68 & 6.19 & -2.91 & $86 \pm 5$ \\
\hline $3-$ HK & 40 & 5.01 & 6.30 & 5.59 & 2.71 & $5 \pm 2$ \\
& 400 & 4.80 & 12.40 & 8.80 & 8.35 & $7 \pm 2$ \\
& 800 & 4.26 & 12.80 & 6.54 & 9.43 & $11 \pm 2$ \\
\hline 5-HPT & 60 & 1.71 & -12.89 & 8.42 & 3.99 & $42 \pm 5$ \\
& 600 & 7.45 & -2.61 & 6.17 & 6.64 & $46 \pm 3$ \\
& 1200 & 7.60 & 0.09 & 6.40 & 8.06 & $53 \pm 8$ \\
\hline XA & 80 & 5.84 & -0.70 & 12.57 & -1.99 & $91 \pm 14$ \\
& 800 & 5.77 & -6.59 & 3.41 & -4.21 & $103 \pm 10$ \\
& 1600 & 4.45 & -8.26 & 3.90 & 1.77 & $100 \pm 19$ \\
\hline I-3CA & 200 & 2.32 & 7.67 & 8.14 & 8.61 & $102 \pm 7$ \\
& 2000 & 7.90 & 1.57 & 6.34 & -3.47 & $101 \pm 6$ \\
& 4000 & 4.38 & 0.40 & 11.86 & 1.72 & $106 \pm 6$ \\
\hline KA & 300 & 6.66 & 6.76 & 4.44 & 4.75 & $85 \pm 34$ \\
& 3000 & 5.52 & 1.13 & 3.98 & 1.50 & $84 \pm 31$ \\
& 6000 & 2.66 & 2.53 & 3.33 & -1.57 & $95 \pm 29$ \\
\hline 5-HIAA & 800 & 2.42 & 11.91 & 3.24 & 7.92 & $95 \pm 6$ \\
& 8000 & 2.34 & 6.22 & 8.99 & -4.07 & $94 \pm 5$ \\
& 16000 & 3.95 & -4.86 & 12.54 & -8.53 & $106 \pm 5$ \\
\hline
\end{tabular}


Table A4. Precision, accuracy and matrix effect at low, medium and high QC levels in mouse urines

\begin{tabular}{|c|c|c|c|c|c|c|}
\hline \multirow{2}{*}{ Metabolites } & \multirow{2}{*}{$\begin{array}{l}\text { Concentration } \\
\qquad(\mathrm{nmol} / \mathrm{L})\end{array}$} & \multicolumn{2}{|c|}{ Intra-day $(n=10)$} & \multicolumn{2}{|c|}{ Inter-day $(n=5)$} & \multirow{2}{*}{$\begin{array}{c}\begin{array}{c}\text { Matrix effect } \\
(n=10)\end{array} \\
\text { Recovery }(\%) \pm s d\end{array}$} \\
\hline & & $\begin{array}{l}\text { CV } \\
(\%)\end{array}$ & $\begin{array}{c}\text { Accuracy } \\
(\%)\end{array}$ & $\begin{array}{l}\mathrm{CV} \\
(\%)\end{array}$ & $\begin{array}{c}\text { Accuracy } \\
(\%)\end{array}$ & \\
\hline \multirow[t]{3}{*}{ 3-HK } & 120 & 3.1 & 7.3 & 5.3 & -4.9 & $102.6 \pm 4$ \\
\hline & 600 & 6.0 & 7.7 & 2.1 & -3.3 & $93.5 \pm 6$ \\
\hline & 1200 & 2.7 & 8.4 & 7.6 & -7.9 & $94.4 \pm 2$ \\
\hline \multirow[t]{3}{*}{ Piconilic acid } & 400 & 2.7 & -11.1 & 10.9 & -4.3 & $123 \pm 13$ \\
\hline & 2000 & 6.0 & -2.9 & 8.4 & -3.3 & $137 \pm 7$ \\
\hline & 4000 & 3.6 & -0.5 & 7.8 & -4.3 & $139 \pm 3$ \\
\hline \multirow[t]{3}{*}{ QA } & 1200 & 19.7 & -15.2 & 11.6 & -0.9 & $47.9 \pm 6.6$ \\
\hline & 6000 & 7.0 & -5.1 & 3.7 & 1.8 & $64.7 \pm 2.4$ \\
\hline & 12000 & 5.4 & -7.1 & 3.0 & -5.2 & $63 \pm 2.3$ \\
\hline \multirow[t]{3}{*}{$5-\mathrm{HT}$} & 160 & 4.1 & -6.8 & 3.2 & -8.3 & $85 \pm 2$ \\
\hline & 800 & 7.7 & -3.3 & 7.1 & -7.7 & $85.6 \pm 6.5$ \\
\hline & 1600 & 3.0 & -0.6 & 13.1 & -5.7 & $85 \pm 3$ \\
\hline \multirow[t]{3}{*}{ 5-HPT } & 200 & 4.8 & 5.3 & 5.6 & 3.9 & $103 \pm 3$ \\
\hline & 1000 & 7.8 & 2.1 & 5.2 & 3.8 & $101.5 \pm 6$ \\
\hline & 2000 & 7.6 & 8.4 & 12.3 & 5.9 & $102 \pm 3.5$ \\
\hline \multirow[t]{3}{*}{ Kyn } & 200 & 4.9 & -10.5 & 9.3 & -2.7 & $103 \pm 3.5$ \\
\hline & 1000 & 8.4 & -10.3 & 6.8 & -0.8 & $103 \pm 6$ \\
\hline & 2000 & 4.4 & -5.9 & 6.5 & -5.7 & $104 \pm 2$ \\
\hline \multirow[t]{3}{*}{$3-\mathrm{HAA}$} & 400 & 1.5 & -0.4 & 2.1 & 1.7 & $103.5 \pm 4$ \\
\hline & 2000 & 5.5 & 0.6 & 3.3 & 3.8 & $103 \pm 5.5$ \\
\hline & 4000 & 2.5 & 3.7 & 5.9 & 0.5 & $104 \pm 1.5$ \\
\hline \multirow[t]{3}{*}{ TA } & 400 & 3.6 & -3.0 & 4.4 & -1.9 & $96 \pm 1$ \\
\hline & 2000 & 5.6 & -3.8 & 4.2 & -1.1 & $93.6 \pm 5.5$ \\
\hline & 4000 & 3.6 & -2.0 & 7.6 & -3.1 & $94.4 \pm 2$ \\
\hline \multirow[t]{3}{*}{ Trp } & 2 & 6.4 & 6.6 & 5.4 & 6.6 & $104.5 \pm 3$ \\
\hline & 10 & 7.1 & 1.5 & 4.7 & 3.2 & $101.5 \pm 6$ \\
\hline & 20 & 3.1 & -0.3 & 5.5 & -5.1 & $101 \pm 2$ \\
\hline \multirow[t]{3}{*}{ 5-HIAA } & 2 & 3.3 & -1.7 & 4.1 & 3.0 & $102.5 \pm 2$ \\
\hline & 10 & 7.3 & -2.2 & 4.3 & 0.0 & $94.5 \pm 6$ \\
\hline & 20 & 3.5 & 2.8 & 6.7 & 0.2 & $94.5 \pm 1.5$ \\
\hline \multirow[t]{3}{*}{$3-1 S$} & 6 & 7.2 & -6.1 & 9.0 & 2.3 & $92 \pm 4$ \\
\hline & 30 & 6.2 & -3.3 & 5.2 & 3.5 & $95.4 \pm 5$ \\
\hline & 60 & 3.3 & -3.3 & 4.0 & -2.1 & $95.6 \pm 2$ \\
\hline \multirow[t]{3}{*}{ N-AS } & 20 & 5.2 & -2.3 & 2.2 & -1.7 & $100 \pm 3$ \\
\hline & 200 & 7.1 & 4.1 & 3.0 & 2.9 & $90.5 \pm 6$ \\
\hline & 400 & 5.5 & 5.5 & 13.3 & 2.7 & $90 \pm 2.5$ \\
\hline \multirow[t]{3}{*}{$X A$} & 2 & 1.5 & -3.1 & 1.1 & -2.1 & $101 \pm 2$ \\
\hline & 10 & 4.4 & 0.9 & 1.7 & 2.3 & $101 \pm 4$ \\
\hline & 20 & 2.6 & 2.5 & 2.4 & 1.7 & $99 \pm 2$ \\
\hline IAM & 40 & 6.3 & 2.1 & 4.1 & -3.3 & $100 \pm 3.5$ \\
\hline
\end{tabular}




\begin{tabular}{|c|c|cc|cc|c|} 
& 200 & 7.3 & 7.2 & 12.6 & 4.9 & $93.5 \pm 5.5$ \\
& 400 & 6.1 & 7.1 & 13.3 & 0.4 & $93 \pm 3$ \\
\hline KA & 2 & 1.0 & -1.4 & 6.1 & 3.4 & $103 \pm 1.5$ \\
& 10 & 5.7 & -0.5 & 5.4 & 2.1 & $100 \pm 6$ \\
& 20 & 3.4 & 1.5 & 7.0 & -1.9 & $101 \pm 2$ \\
\hline ILA & 200 & 4.7 & 6.1 & 5.1 & 9.2 & $104 \pm 6$ \\
& 1000 & 5.8 & 1.6 & 4.2 & 3.6 & $96 \pm 5$ \\
& 2000 & 2.1 & 3.8 & 3.0 & 0.5 & $95.5 \pm 1$ \\
\hline I-3CA & 200 & 7.9 & 4.2 & 5.6 & 5.0 & $18 \pm 2$ \\
& 1000 & 6.7 & 3.1 & 2.8 & 3.3 & $64.5 \pm 4$ \\
& 2000 & 2.3 & 5.1 & 9.0 & -0.3 & $77 \pm 1.5$ \\
\hline IAA & 1200 & 3.5 & 3.0 & 4.9 & 4.9 & $93.5 \pm 3$ \\
& 6000 & 6.2 & 1.0 & 6.9 & -0.5 & $93.5 \pm 5.5$ \\
& 12000 & 2.4 & 3.2 & 8.1 & -1.6 & $92 \pm 1.5$ \\
\hline Tryptophol & 8 & 18.9 & 16.9 & 25.8 & -8.5 & $84.5 \pm 20$ \\
& 40 & 7.7 & 14.4 & 9.7 & 9.9 & $95.5 \pm 5$ \\
& 80 & 3.9 & -24.9 & 11.2 & 3.5 & $90.5 \pm 3$ \\
\hline Melatonin & 8 & 3.1 & 7.3 & 4.4 & 1.0 & $96 \pm 3$ \\
& 40 & 6.0 & 7.7 & 4.9 & 6.3 & $91.5 \pm 5$ \\
& 80 & 2.7 & 8.4 & 9.3 & 4.4 & $91.5 \pm 1$ \\
\hline
\end{tabular}


Table A5. Precision, accuracy and matrix effect at low, medium and high QC levels in mouse intestinal contents

\begin{tabular}{|c|c|c|c|c|c|c|}
\hline \multirow{2}{*}{ Metabolites } & \multirow{2}{*}{$\begin{array}{l}\text { Concentration } \\
\text { (nmol/mL) }\end{array}$} & \multicolumn{2}{|c|}{ Intra-day $(n=10)$} & \multicolumn{2}{|c|}{ Inter-day $(n=5)$} & \multirow{2}{*}{$\begin{array}{c}\begin{array}{c}\text { Matrix effect } \\
(n=10)\end{array} \\
\text { Recovery }(\%) \pm s d\end{array}$} \\
\hline & & $\begin{array}{l}\mathrm{CV} \\
(\%)\end{array}$ & $\begin{array}{c}\text { Accuracy } \\
\text { (\%) }\end{array}$ & $\begin{array}{l}\text { CV } \\
\text { (\%) }\end{array}$ & $\begin{array}{c}\text { Accuracy } \\
(\%)\end{array}$ & \\
\hline \multirow[t]{3}{*}{ 3-HAA } & 40 & 9.98 & 6.80 & 5.49 & 1.84 & $16 \pm 6$ \\
\hline & 400 & 13.77 & 1.28 & 6.98 & 7.06 & $128 \pm 53$ \\
\hline & 800 & 15.26 & -3.81 & 12.63 & -3.31 & $114 \pm 77$ \\
\hline \multirow[t]{3}{*}{ 3-HK } & 20 & 19.65 & 11.13 & 39.71 & 24.55 & $11 \pm 2$ \\
\hline & 200 & 5.52 & 6.24 & 34.46 & 21.01 & $17 \pm 5$ \\
\hline & 400 & 7.62 & 2.15 & 38.60 & 18.08 & $19 \pm 4$ \\
\hline \multirow[t]{3}{*}{$3-1 S^{*}$} & 200 & 12.97 & 8.81 & 10.40 & 19.89 & $132 \pm 11$ \\
\hline & 2000 & 8.07 & -1.75 & 3.36 & -0.62 & $108 \pm 1$ \\
\hline & 4000 & 4.79 & -1.20 & 1.90 & 1.78 & $111 \pm 2$ \\
\hline \multirow[t]{3}{*}{ 5-HIAA } & 800 & 1.47 & 14.24 & 9.35 & 2.23 & $50 \pm 2$ \\
\hline & 8000 & 1.81 & 0.36 & 7.98 & 2.99 & $49 \pm 2$ \\
\hline & 16000 & 7.28 & -11.87 & 10.10 & -4.85 & $48 \pm 3$ \\
\hline \multirow[t]{3}{*}{ 5-HPT } & 40 & 36.70 & -13.56 & 9.85 & 13.18 & $40 \pm 2$ \\
\hline & 400 & 7.07 & -2.83 & 2.43 & 3.95 & $39 \pm 2$ \\
\hline & 800 & 9.60 & -3.46 & 3.13 & 1.74 & $39 \pm 2$ \\
\hline \multirow[t]{3}{*}{$5-\mathrm{HT}^{*}$} & 300 & 6.90 & 16.60 & 9.98 & 7.37 & $24 \pm 1$ \\
\hline & 3000 & 4.07 & -10.19 & 5.75 & -7.14 & $26 \pm 3$ \\
\hline & 6000 & 11.39 & -17.86 & 6.10 & -10.53 & $26 \pm 2$ \\
\hline \multirow[t]{3}{*}{ I-3CA } & 300 & 2.24 & 16.87 & 9.37 & 7.43 & $51 \pm 3$ \\
\hline & 3000 & 3.97 & 10.49 & 7.09 & 3.14 & $54 \pm 2$ \\
\hline & 6000 & 7.74 & 2.87 & 7.29 & -1.31 & $57 \pm 1$ \\
\hline \multirow[t]{3}{*}{ IAA* } & 100 & 8.27 & -3.44 & 4.49 & 5.35 & $49 \pm 2$ \\
\hline & 1000 & 2.86 & -6.76 & 4.83 & 1.96 & $50 \pm 2$ \\
\hline & 2000 & 8.58 & -16.75 & 8.69 & -3.77 & $49 \pm 2$ \\
\hline \multirow[t]{3}{*}{ IAM } & 4 & 8.27 & 6.84 & 3.85 & 9.59 & $53 \pm 8$ \\
\hline & 40 & 8.63 & 5.37 & 5.09 & 5.09 & $50 \pm 2$ \\
\hline & 80 & 10.02 & 5.55 & 6.53 & 30.55 & $47 \pm 5$ \\
\hline \multirow[t]{3}{*}{ ILA* } & 300 & 5.07 & 1.91 & 2.55 & 3.29 & $55 \pm 4$ \\
\hline & 3000 & 4.76 & -4.52 & 6.97 & 3.00 & $56 \pm 3$ \\
\hline & 6000 & 11.44 & -13.67 & 14.85 & -0.28 & $57 \pm 2$ \\
\hline \multirow[t]{3}{*}{ KA } & 120 & 5.73 & 13.64 & 6.62 & 10.48 & $51 \pm 3$ \\
\hline & 1200 & 3.46 & 8.12 & 4.33 & 3.91 & $50 \pm 2$ \\
\hline & 2400 & 7.33 & 3.87 & 5.65 & -1.47 & $50 \pm 3$ \\
\hline \multirow[t]{3}{*}{ Kyn* } & 100 & 19.77 & 3.79 & 29.64 & 13.10 & $57 \pm 3$ \\
\hline & 1000 & 6.45 & 6.86 & 7.89 & 4.07 & $56 \pm 2$ \\
\hline & 2000 & 8.77 & -0.52 & 1.19 & 1.35 & $56 \pm 3$ \\
\hline \multirow[t]{3}{*}{ Melatonin } & 4 & 2.22 & 1.66 & 4.11 & 0.24 & $69 \pm 5$ \\
\hline & 40 & 2.69 & -3.03 & 2.56 & -3.00 & $70 \pm 3$ \\
\hline & 80 & 7.07 & -4.99 & 2.53 & -3.89 & $67 \pm 4$ \\
\hline
\end{tabular}




\begin{tabular}{|c|c|cc|cc|c|}
\hline N-AS & 20 & 6.26 & 5.13 & 7.31 & 1.48 & $79 \pm 7$ \\
& 200 & 8.09 & 6.46 & 7.32 & 6.41 & $71 \pm 5$ \\
& 400 & 11.01 & 2.67 & 7.45 & 0.80 & $67 \pm 4$ \\
\hline Piconilic acid* & 400 & 7.37 & 11.70 & 16.4 & 17.00 & $100 \pm 10$ \\
& 4000 & 6.29 & 0.80 & 3.8 & -1.04 & $104 \pm 5$ \\
& 8000 & 4.79 & -4.38 & 2.2 & -3.22 & $99 \pm 3$ \\
\hline QA & 60 & 9.84 & 8.17 & 12.8 & 11.89 & $93 \pm 17$ \\
& 600 & 8.35 & -7.09 & 4.5 & 0.32 & $89 \pm 6$ \\
& 1200 & 4.13 & -12.20 & -0.6 & -7.54 & $82 \pm 2$ \\
\hline TA & 60 & 10.65 & 7.95 & 8.63 & -8.45 & $34 \pm 4$ \\
& 600 & 4.50 & -3.71 & 6.80 & -3.08 & $43 \pm 3$ \\
& 1200 & 7.12 & -6.87 & 6.09 & -2.22 & $46 \pm 4$ \\
\hline Trp* & 2 & 3.5 & 3.0 & 4.5 & -0.21 & $106 \pm 4$ \\
& 20 & 6.2 & 1.0 & -5.5 & -11.57 & $101 \pm 3$ \\
& 40 & 2.4 & 3.2 & -7.6 & -5.43 & $100 \pm 2$ \\
\hline Tryptophol & 100 & 18.9 & 16.9 & 14.22 & 3.54 & $48 \pm 6$ \\
& 1000 & 7.7 & 14.4 & 8.91 & 9.18 & $60 \pm 2$ \\
& 2000 & 3.9 & -24.9 & 7.72 & 6.53 & $60 \pm 2$ \\
\hline XA & 80 & 3.1 & 7.3 & 4.27 & 3.47 & $59 \pm 9$ \\
& 800 & 6.0 & 7.7 & 2.47 & 1.09 & $53 \pm 12$ \\
& 1600 & 2.7 & 8.4 & 7.33 & -4.48 & $54 \pm 9$ \\
\hline
\end{tabular}


Fig. A1. Mouse metabolite concentrations in various matrices (in $\mu \mathrm{mol} / \mathrm{L}$ for urine and serum; in $\mu \mathrm{mol} / \mathrm{L} / \mathrm{g}$ for lyophilized cecum, intestinal and liver freeze-dried contents).
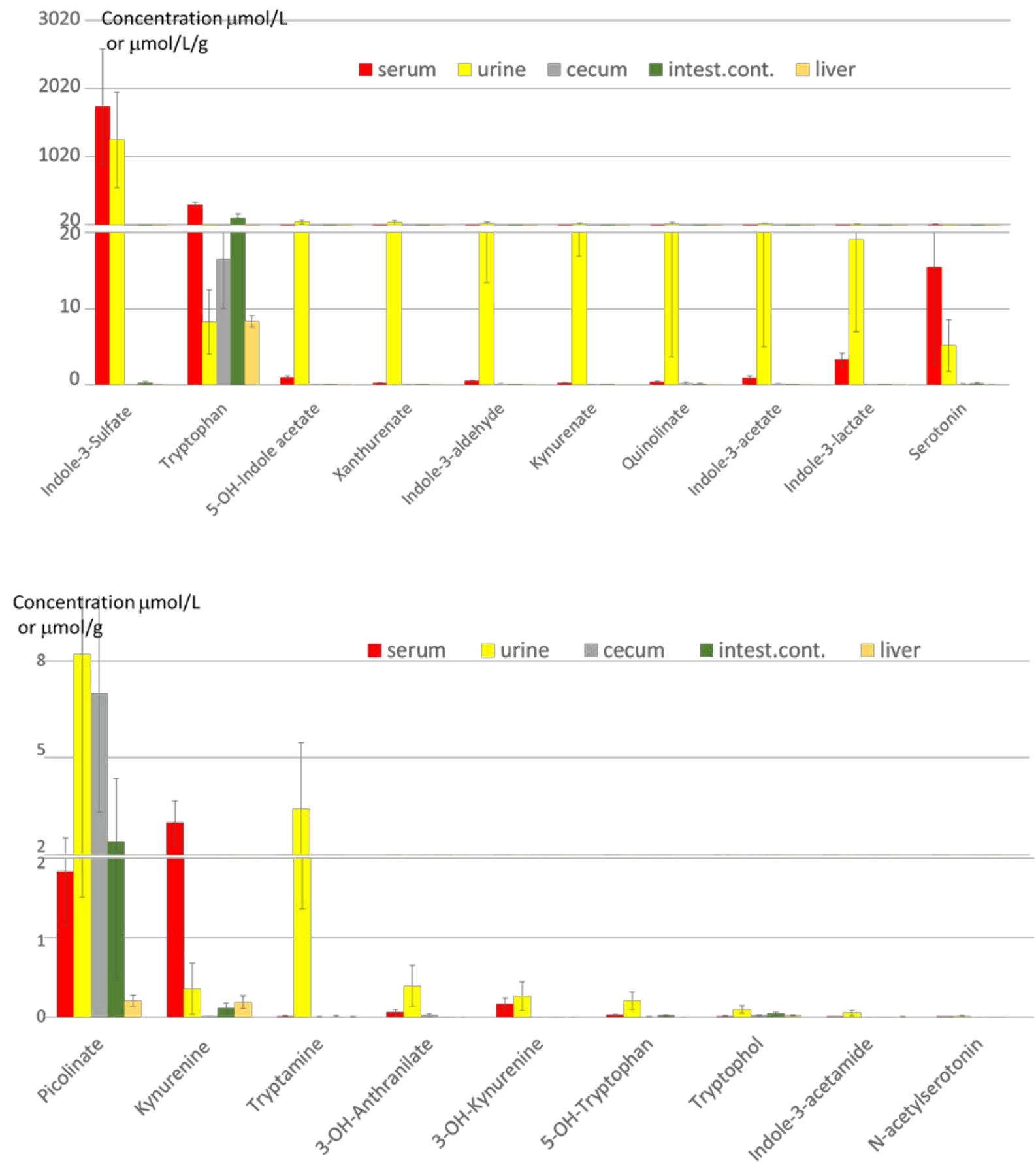
Fig. A2. Rank -Higher to lower concentration intensity of a metabolite in mice matrices. The most intense metabolite was classified with a score of 20 .

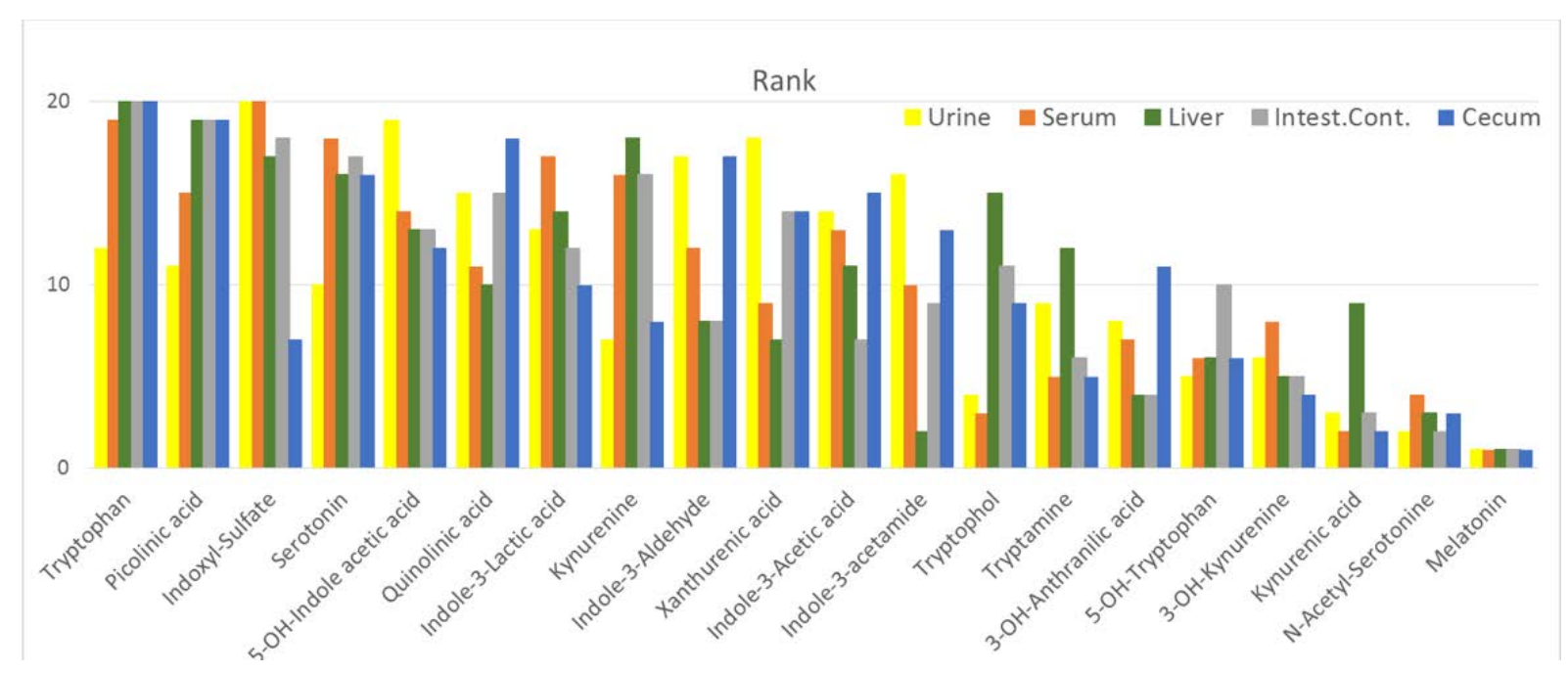

